\title{
Des migrants et des mots : Une analyse numérique des débats médiatiques sur les migrations et l'environnement
}

of Migrants and Words. A Web-Based Analysis of Online Debates on Migrations and Environment

Tommaso Venturini, François Gemenne et Marta Severo

\section{(2) OpenEdition Journals}

\section{Édition électronique}

URL : http://journals.openedition.org/conflits/18594

DOI : 10.4000/conflits. 18594

ISSN : $1777-5345$

\section{Éditeur :}

CCLS - Centre d'études sur les conflits lilberté et sécurité, L'Harmattan

\section{Édition imprimée}

Date de publication : 30 décembre 2012

Pagination : 133-156

ISBN : 978-2-343-00589-8

ISSN : 1157-996X

\section{Référence électronique}

Tommaso Venturini, François Gemenne et Marta Severo, « Des migrants et des mots : Une analyse numérique des débats médiatiques sur les migrations et l'environnement », Cultures \& Conflits [En ligne], 88 | hiver 2012, mis en ligne le 15 mars 2014, consulté le 30 mars 2021. URL : http:// journals.openedition.org/conflits/18594; DOI : https://doi.org/10.4000/conflits.18594 


\section{Des migrants et des mots : Une analyse numérique des débats médiatiques sur les migrations et l'environnement}

\section{Tommaso VENTURINI, François GEMENNE, Marta SEVERO}

Tommaso Venturini est chercheur et professeur associé à Sciences Po où il coordonne les activités de recherche du médialab. Ses recherches se concentrent sur les méthodes numériques et quali-quantitatives pour les sciences sociales. Il enseigne et coordonne plusieurs projets sur la cartographie des controverses.

François Gemenne est chercheur en science politique (FNRS/CEDEM - ULg/Iddri - Sciences Po). Ses recherches sont principalement consacrées aux migrations et aux déplacements de populations liés aux changements de l'environnement, ainsi qu'aux politiques d'adaptation au changement climatique. Il enseigne également ces questions à Sciences Po, à l'Université de Paris 13 et à l'Université Libre de Bruxelles.

Marta Severo est maître de conférences en Communication auprès du laboratoire Geriico de l'Université de Lille 3. Elle travaille sur les thèmes de la communication et de la gestion du patrimoine culturel et notamment sur la contribution des technologies numériques. Elle est l'auteur de Heritage Networks. Managing Network Cultural Heritage with the Web (2009).

Vous savez, il y a un débat ici au sujet des réfugiés. Laissez-moi vous dire quelle est ma position et celle des personnes autour de cette table : les personnes dont nous parlons ne sont pas des réfugiés. Ce sont des Américains, et ils méritent l'aide, l'amour et la compassion de nos compatriotes ${ }^{1}$.

Président George W. Bush, à propos des populations déplacées par l'ouragan Katrina, 6 septembre 2005

1. «You know, there's a debate here about refugees. Let me tell you my attitude and the atti- 


\section{L’intérêt d'une controverse}

Jouragan Katrina, qui ravagea les côtes du Golfe du Mexique en août L 2005, provoqua l'évacuation de plus d'un million de personnes. Certains trouvèrent refuge chez des proches, d'autres dans des centres d'accueil mis en place par les autorités ou des organisations caritatives. Très rapidement, les médias allaient jouer un rôle central dans la caractérisation des victimes de la catastrophe. Immédiatement après la catastrophe, le terme qui était alors le plus fréquemment utilisé pour désigner les évacués était celui de « réfugiés de Katrina » 2 . Le terme, néanmoins, fut immédiatement rejeté par ceux qu'il prétendait désigner. Ceux-ci insistèrent pour être appelés « évacués », ou « survivants », mais surtout pas « réfugiés ». Pour beaucoup, être appelé(e) « réfugié » revenait à être considéré comme un citoyen de seconde zone, un étranger dans son propre pays ${ }^{3}$. Refuser cette étiquette était aussi une manière de rappeler à leur propre État ses obligations envers ses citoyens. L'ancien maire de La Nouvelle-Orléans, Marc Morial, proposa l'emploi du terme "citoyens réfugiés ", sans plus de succès. Après que les victimes se furent indignées contre l'emploi du terme de "réfugiés ", la plupart des médias cessèrent de l'utiliser et le remplacèrent par d'autres termes, notamment celui d' «évacués ». Mais d'autres médias, et notamment CNN, le New York Times et l'Associated Press, persistèrent à l'utiliser. Le 7 septembre 2005, un des présentateurs-vedettes de CNN, Lou Dobbs, justifiait ainsi à l'antenne l'emploi du terme :

Au fait, vous avez entendu dans cette émission plusieurs personnes, dont le Révérend Jesse Jackson, nous intimer de ne pas utiliser le terme de "réfugiés » pour décrire les citoyens de La NouvelleOrléans qui avaient fui leur maison. Jackson et d'autres, notamment le Président Bush, ont dit ou sous-entendu que ce terme était racialement connoté. Le dictionnaire Miriam Webster définit un réfugié comme quelqu'un qui fuit. [...] Le Président, Jackson et d'autres ont l'air de penser que les médias ont créé le terme de réfugié dans le seul but de décrire les victimes de l'ouragan Katrina. C'est n'importe quoi. Même une revue rapide de la couverture médiatique de catastrophes comme l'ouragan Andrew, des inondations dans le Midwest en 1993 ou des feux de forêts dans l'Ouest fera apparaître l'usage du terme « réfugié » par les médias. Je suis fier de vous dire

tude of people around this table: The people we're talking about are not refugees. They are Americans, and they need the help and love and compassion of our fellow citizens. » (traduction des auteurs)

2. Sommers S. R., Apfelbaum E. P., Dukes K. N., Toosi N., et Wang E. F., "Race and Media Coverage of Hurricane Katrina: Analysis, Implications, and Future Research Questions", Analyses of Social Issues and Public Policy, 6, 2006, p. 40.

3. Gemenne F., "What's in a name: social vulnerabilities and the refugee controversy in the wake of hurricane Katrina", in Jäger J., Afifi T. (eds.), Environment, Forced Migration and Social Vulnerability, Berlin, Springer, 2010. 
que cette chaîne a résisté aux appels de ceux qui lui disaient quels mots employer. A même rejeté, en fait, la suggestion des Nations Unies que nous utilisions, plutôt que « réfugiés », l'expression « déplacés internes ». J’adore celle-là. Nous continuerons à utiliser le terme dans cette émission quand nous pensons que c'est le plus approprié ${ }^{4}$.

Outre la colère des victimes, d'autres raisons furent avancées pour rejeter l'emploi du terme "réfugiés » : d'aucuns estimaient que le terme retirait aux victimes leur dignité 5 , tandis que d'autres, tels les Révérends Jackson et Sharpton, voyaient dans le terme une preuve évidente de discrimination raciale. Le linguiste Geoffrey Nunberg, dans une étude postée sur son site personnel, montre ainsi que le terme « réfugié » était deux fois plus employé que le terme "évacué » quand il était employé en conjonction avec les termes « noir » ou « pauvre» 6 .

Pourquoi faire état ici de cette controverse ? D’abord parce qu'elle illustre la catastrophe sociale qu'a été Katrina, et les profondes inégalités sociales que l'ouragan a mises à jour ${ }^{7}$. Ensuite parce qu'elle révèle un vide catégoriel. Aucun terme, aujourd'hui, ne fait l'unanimité pour désigner ceux qui sont déplacés en raison de dégradations de leur environnement. Et s'il n'existe aucun terme consensuel, c'est avant tout parce que la réalité des migrations liées à l'environnement est polymorphe : certain(e)s sont forcé(e)s de quitter leur domicile à la suite de catastrophes naturelles brutales, tandis que d'autres font le choix de partir, à la recherche d'un environnement plus clément. Certain(e)s partent très loin, d'autres ne peuvent se déplacer qu'à quelques kilomètres. Certain(e)s peuvent revenir chez eux après quelques semaines ou quelques années, tandis que d'autres ne reviennent jamais. La relation entre changements environnementaux et mouvements migratoires est à ce point

4. "You've heard on this broadcast, by the way, several people, including Reverend Jesse Jackson and others admonish us not to use the term refugee when describing the New Orleans citizens who have had to flee their homes. Jackson and others including President Bush have said or implied that term is racially insensitive. In my opinion, straightforwardly, Reverend Jackson and President Bush are not entirely correct. The Miriam Webster Dictionary defines refugee as one who flees. (...) The president, Jackson and others apparently think that news organizations created the term refugee just to describe victims of Hurricane Katrina. Hardly. Even a cursory review of reporting of such disaster of Hurricane Andrew, the 1993 midwestern floods and wildfires through the West have all prompted the use of the term refugee by news organizations. I'm proud to tell you that this network has resisted others telling them how to use words. Rejecting, in fact, the United Nations suggestion that we use, instead of refugee, the expression "internally displaced persons". I love that one. We'll continue here to use the term on this broadcast where we think it is most descriptive » (traduction des auteurs). Dans «Lou Dobbs tonight », CNN, 7 septembre 2005.

5. Masquelier A., "Why Katrina's Victims Aren't Refugees: Musings on a 'Dirty' Word”, American Anthropologist, 108, 4, 2006, pp. 735-743

6. Nunberg G., "When Words Break Down", 2005. http://people.ischool.berkeley.edu/ nunberg/looting.html (consulté le 20 décembre 2012).

7. Huret R., Katrina, 2005. L'ouragan, l'État et les pauvres aux États-Unis, Paris, Éditions de l'EHESS, 2010. 
complexe et multiforme qu'il est sans doute impossible de trouver un seul terme qui en fasse la synthèse, et qui soit à même de refléter la diversité des vécus des migrants ${ }^{8}$.

Pourtant les termes que l'on emploie pour désigner cette réalité comptent, et la controverse de Katrina est là pour nous le rappeler. Depuis les travaux de Becker ${ }^{9}$ et Goffman ${ }^{10}$ sur la théorie de l'étiquetage, les chercheurs en sciences sociales savent bien que les termes employés impliquent une catégorisation, et que cette catégorisation forge l'identité de ceux qu'elle désigne. Alors que de nombreux travaux existent sur les conséquences de la catégorisation des réfugiés sur leur identité personnelle et les réponses politiques qui sont déployées ${ }^{11}$, peu de travaux similaires existent pour la relation environnement-migration, malgré la grande variété de vocables utilisés pour désigner un même phénomène. Pourtant, les mots comptent : à chaque terme est associée une valeur symbolique, qui peut à la fois affecter la vulnérabilité de ceux qu'il désigne, mais également déterminer les réponses politiques qui seront mises en œuvre pour encadrer ces migrations. Ainsi, plusieurs études ont montré combien ces questions identitaires étaient déterminantes dans la capacité d'adaptation de ceux qui étaient touchés par des changements environnementaux ${ }^{12}$. Mais rares sont les travaux de réflexion et d'analyse sur les termes employés pour désigner ceux qui migraient pour des raisons environnementales ${ }^{13}$. Seuls les termes de "réfugié climatique » ou de "réfugié environnemental » se sont attirés les foudres des juristes, ceux-ci relevant généralement que le terme de "réfugié » était strictement défini par la Convention de Genève de 1951, dont ne relevaient pas ceux qui migraient pour des raisons environnementales ${ }^{14}$.

Dans la communauté académique, les débats sur les termes à employer sont vifs, et recoupent largement les débats qui touchent à la définition des migrations liées à l'environnement, et sur la protection à accorder aux migrants. Ainsi, il est possible d'identifier plusieurs tendances dans la littérature. En premier lieu, certains auteurs et organisations militent pour une reconnaissance officielle du statut de « réfugié environnemental » ou de « réfu-

8. Piguet E., Pécoud A., et de Guchteneire P., "Migration and Climate Change: An Overview", Refugee Survey Quarterly, 30, 3, 2011, pp. 1-23.

9. Becker H., Outsiders: Studies in the Sociology of Deviance, New York, Free Press, 1963.

10. Goffman E., Stigma: Notes on the Management of Spoiled Identity, Englewood Cliffs, N.J., Prentice-Hall, 1963.

11. Zetter R., "Labelling Refugees: Forming and Transforming a Bureaucratic Identity", Journal of Refugee Studies, 4, 1991, pp. 39-62.

12. Farbotko C., "Tuvalu and climate change: Constructions of environmental displacement in the Sydney Morning Herald", Geografiska Annaler, 87, B, 2005, pp. 279-293.

13. Dun O., Gemenne F., "Defining 'environmental migration”, Forced Migration Review, 31, 2008, pp. 10-11,

14. McAdam J., "Swimming against the Tide: Why a Climate Change Displacement Treaty is Not the Answer", International Journal of Refugee Law, 23, 2011, pp. 2-27 ; Cournil C., "Vers une reconnaissance des 'réfugiés écologiques" ? Quelle(s) protection(s), quel(s) statut(s) ? », Revue du Droit Public et de la Science Politique, 4, 2006, pp. 1035-1066. 
gié climatique » dans le droit international, en soulignant le vide juridique qui entoure leur protection. Logiquement, ces auteurs emploieront volontiers le terme de "réfugié ", précisément pour insister sur l'absence de critères environnementaux dans la définition officielle du concept de « réfugié » de la Convention de Genève de $1951^{15}$. La plupart de ces auteurs souhaitent dès lors soit que la Convention de Genève soit révisée pour inclure des critères environnementaux, soit qu'une nouvelle convention soit adoptée pour garantir à ces personnes une protection dans le droit international.

A l'inverse, d'autres auteurs estiment que l'emploi du terme de "réfugiés » est particulièrement préjudiciable, et qu'une protection spécifique dans le droit international ne serait pas appropriée, voire contre-productive. Logiquement, ces auteurs condamnent l'emploi du terme de "réfugiés », et lui préféreront plus volontiers le terme de "déplacés»16. Certains auteurs contestent également l'idée qu'il soit possible d'identifier une catégorie spécifique de migrants dont la migration soit liée à l'environnement, et insistent plutôt sur la multi-causalité des migrations, et l'impossibilité d'isoler les facteurs environnementaux parmi d'autres facteurs de migration. Ces auteurs éviteront dès lors l'emploi d'un terme spécifique pour désigner ces migrants ${ }^{17}$.

La controverse dépasse largement la sphère académique, puisqu'elle touche à la question de la protection à accorder aux migrants, et donc aussi aux mandats des différentes agences qui pourraient fournir cette protection. À cet égard, il est particulièrement intéressant d'observer le positionnement du Haut Commissariat des Nations Unies pour les Réfugiés (HCR) et de l'Organisation Internationale pour les Migrations (OIM), qui sont les deux principales organisations en charge de ces migrants sur le terrain, sans que cette mission fasse explicitement partie de leur mandat. Soucieux de ne pas introduire de confusion avec son mandat de protection des réfugiés, le HCR préférera ainsi généralement parler de « personnes déplacées pour des raisons

15. Westing A. H., "Environmental refugees: A growing category of displaced persons", Environmental Conservation, 19, 3, 1992, pp. 201-207 ; McCue G. S., "Environmental Refugees: Applying International Environmental Law to Involuntary Migration”, Georgetown International Environmental Law Review, 6, 1, 1993, pp. 151-190 ; Myers N., "Environmental refugees: a growing phenomenon of the 21st century", Philosophical Transactions of the Royal Society, B 357 (1420), 2002, pp. 609-613 ; Conisbee M. et Simms A., Environmental Refugees. The case for Recognition, Londres, New Economics Foundation, 2003 ; Williams A., "Turning the Tide: Recognizing Climate Change Refugees in International Law", Law \& Policy, 30, 4, 2008, pp. 502-529.

16. Kälin W., "Conceptualising Climate-Induced Displacement”, in McAdam J. (ed.) Climate Change and Displacement. Multidisciplinary Perspectives, Oxford, Hart, 2010, pp.81-104; McAdam J., "Swimming against the Tide: Why a Climate Change Displacement Treaty is Not the Answer", op. cit.

17. Kibreab G., "Environmental Causes and Impact of Refugee Movements: A Critique of the Current Debat", Disasters, 21, 1, 1997, pp. 20-38; Jonsson G., The environmental factor in migration dynamics - a review of African case studies, Oxford, International Migration Institute, 21, 2010. 
environnementales » ${ }^{18}$, soulignant ainsi le caractère forcé de leur migration et donc la légitimité du HCR à se saisir de la question - tout en veillant à les distinguer clairement des réfugiés. A l'inverse, l'OIM emploiera plus volontiers le terme de «migrants environnementaux » ${ }^{19}$, dont elle proposera une définition - manière de souligner que l'organisation se trouve bien dans le cadre de son mandat général de protection des migrants.

La controverse sur les termes à employer n'est donc pas simplement affaire de vocabulaire : elle touche à la conceptualisation même des migrations liées à l'environnement, et à la protection de ces migrants, ainsi qu'à la politique qui leur sera appliquée quant à leurs droits fondamentaux ou au franchissement de frontières. L'emploi d'un terme plutôt qu'un autre révélera non seulement une manière différente d'appréhender et de conceptualiser ces mouvements de populations, mais également une indication quant à la manière la plus adéquate d'assurer leur protection. A cet égard, une fracture importante se situe autour de l'emploi du terme de "réfugié », car cette fracture traduit aussi, généralement, un positionnement quant à la nécessité de créer dans le droit international un statut de "réfugié environnemental » ou de « réfugié climatique».

Cet article est l'une des premières tentatives visant à analyser la production de discours sur la relation entre environnement et migrations au travers des termes employés pour désigner les migrants. Jusqu'ici, cette discussion était restée confinée aux débats sur la recherche d'une définition consensuelle, sans que la question de l'usage de ces termes ne soit véritablement abordée. Or, au cours des dernières années, les liens entre changements environnementaux et migrations ont fait l'objet de mobilisations importantes, et ont logiquement généré de nombreux discours médiatiques. Les liens entre changement climatique et migrations sont de plus en plus débattus, non seulement dans les contextes institutionnels de la recherche et des organisations internationales, comme on vient de le voir, mais également dans les discours des citoyens ordinaires. Ce sont ces discours qui constituent l'objet de cet article, et qui sont analysés à l'aide d'une méthode innovante fondée sur une cartographie d'internet ${ }^{20}$.

18. Gorlick B., "Environmentally Displaced Persons: A UNHCR Perspective”, in "Environmental Refugees: the Forgotten Migrants”, Discussion Event, New York, 2007.

19. Laczko F., Aghazarm C., Migration, Environment and Climate Change: Assessing the Evidence, Geneva, International Organization for Migration, 2009.

20. Cet article constitue un premier test des méthodes de recherche d'un plus large projet dédié à la cartographie des controverses sur le changement climatique. Financé par le 7ème programme cadre de l'Union Européenne, le projet EMAPS (Grant Agreement numéro 288964) vise à analyser et à représenter le débat autour des stratégies d'adaptation au réchauffement global (voir http://www.emapsproject.com/ pour plus d'informations). 
Se déployant sous l'impulsion d'un nombre croissant d'acteurs et sous l'élan d'injonctions fortes et souvent opposées, la question du lien entre migrations et environnement se présente volontiers comme un « moteur à controverses ». D’un côté, le phénomène des migrations prend de plus en plus d'ampleur, accru par le fossé grandissant entre pays riches et pays pauvres, et facilité par un meilleur accès aux moyens de transport international. Ce phénomène suscite des tensions toujours plus vives, qui opposent le désir et le droit à la mobilité de millions de personnes aux exigences de contrôle des pays d'origine et de destination. Par ailleurs, depuis quelques années, le changement climatique et ses conséquences ont acquis une beaucoup plus grande visibilité dans le débat international. Avec le changement climatique, ce n'est pas une technologie ou une institution particulière qui est remise en discussion, mais l'organisation même de notre vie collective, des moindres aspects de la vie quotidienne jusqu'aux relations internationales.

Le débat académique et institutionnel, loin d'avoir tranché la question de la définition et de la terminologie, emploie une très grande variété de vocables différents pour caractériser la relation entre environnement et migrations, tandis que les organisations de la société civile utilisent volontiers des images et notions à fort impact émotionnel pour nourrir l'imaginaire du public.

Les contours encore flous de la relation entre environnement et migrations doivent-ils nous conduire à ignorer le débat public sur ce sujet, sous prétexte qu'il s'agirait d'un débat stérile et abstrait, du fait de l'impossibilité d'isoler les causes environnementales des migrations?

$\mathrm{Au}$ contraire, précisément à cause de sa nature de "moteur à controverses », la question du lien entre migrations et environnement nous semble être un sujet extrêmement intéressant. Comme toutes les controverses bien vivantes, ce débat permet d'expliciter et donc d'analyser les lignes de fracture traversant notre vie collective ${ }^{21}$.

Ce débat complexe se cristallise en partie autour de la question de l'étiquette utilisée pour les personnes déplacées par des changements environnementaux. La question est loin d'être purement formelle, puisqu'elle a des conséquences sur le droit international, sur les réponses politiques à mettre en œuvre, mais aussi, plus fondamentalement, sur la manière dont les migrants se perçoivent et dont nous les percevons. En caricaturant à peine, si on les appelle "réfugiés ", cela suppose que leur migration est forcée. Si on les appelle " migrants ", c'est qu'au contraire leur migration est choisie. Si on les appelle "refugiés », on reconnaît implicitement le besoin d'assistance de ces popula-

21. Venturini T., "Diving in Magma: How to Explore Controversies with Actor-Network Theory”, Public Understanding of Science, 20, 4, 2010 ; Venturini T., "Building on Faults: How to Represent Controversies with Digital Méthods”, Public Understanding of Science, (à paraître). 
tions. Si on les appelle " migrants », on a volontiers tendance à les considérer eux-mêmes comme un problème. Si on les appelle « climatiques », on attribue directement la cause de leur migration au réchauffement global. Si on les appelle " environnementaux », la cause de leur déplacement apparaît tout de suite plus vague et incertaine. Et, bien sûr, les acteurs n'hésitent pas à mélanger substantifs et adjectifs, pour obtenir l'exacte nuance qui leur convient.

Dans la discussion sur les étiquettes se trouve au fond condensée toute la controverse sur les migrations liées à l'environnement, et avec elle beaucoup des débats sur les conséquences de la crise écologique.

\section{Méthode}

Dans cet article, nous ne nous intéressons pas directement au débat académique et institutionnel sur le sujet, mais uniquement à ses manifestations médiatiques. Et même, plus précisément, au vocabulaire qui est employé dans ces manifestations médiatiques. Comment ces débats ont-ils investi les sphères de l'opinion publique ? Question difficile, car l'opinion publique est par définition fuyante et hétérogène ${ }^{22}$. Elle ne s'exprime pas dans une arène prédéfinie, elle ne suit aucune cohérence formelle, elle est constituée par un flux de voix diverses, impossible à prévoir et constamment changeant. L'opinion publique est bien sûr composée par les chercheurs, les institutions, les médias, les organisations, mais elle accueille aussi une multitude d'acteurs inattendus (activistes, bloggeurs, savants amateurs, citoyens...) qu'on ne peut ignorer. Comment étudier une telle cacophonie, et comment l'appréhender ?

Internet nous aide dans ce travail car il offre un espace assez neutre pour que toutes ces voix s'y expriment, mais suffisamment stable, techniquement, pour qu'on puisse toutes les enregistrer. Mais le Web est vaste et mal indexé : comment y retrouver les discours qu'on y cherche ? Demandez à n'importe quel expert en information mining comment s'y prendre pour trouver l'aiguille dans la botte de foin : il vous répondra qu'il faut surtout avoir la bonne query, la bonne requête, la combinaison magique qui permet d'identifier les informations qu'on cherche et de filtrer le bruit qui les entoure. Or, la discussion sur le vocabulaire des migrations liées à l'environnement nous offre exactement cela : une bonne requête ou - encore mieux - une série de bonnes requêtes capables d'identifier les différents discours sur ces migrations.

Les cartes que nous allons discuter dans les prochaines pages n'ont pas été produites de manière automatique. Trop souvent, lorsqu'on parle de méthodes numériques pour les sciences sociales ${ }^{23}$ ou de computational social sciences ${ }^{24}$, on imagine des algorithmes fantastiques qui permettraient d'ap-

22. Lippmann W., The Phantom Public, New York, The Macmillan Company, 1927.

23. Rogers R., Information Politics on the Web, Cambridge, Mass, MIT Press, 2004 et Rogers R. The End of the Virtual: Digital Methods, Amsterdam, Amsterdam University Press, 2009. 
puyer sur un bouton et d'obtenir automatiquement un résultat. La réalité est tout autre. Les méthodes numériques demandent une intervention continue du chercheur, et sont jalonnées par des centaines de choix subjectifs qui ne peuvent s'appuyer que sur l'expertise de l'analyste ${ }^{25}$.

Dans les paragraphes suivants, nous procéderons à une description très détaillée des passages qui nous ont permis d'arriver aux cartes que nous allons analyser. Avant de nous lancer dans les détails méthodologiques, il est toutefois important de donner au lecteur une idée d'ensemble de l'approche employée dans cet article. Au delà de ses technicités, notre démarche est relativement linéaire. Il s'agit de s'appuyer sur Google (aujourd'hui le plus influent des moteurs de recherche) pour collecter un ensemble de pages web utilisant les diverses étiquette associées aux migrations climatiques. Sur la base de ces corpus, nous avons ensuite analysé quelles expressions lexicales apparaissaient le plus fréquemment dans les pages associées à l'une ou à l'autre étiquette. Nous avons enfin visualisé les relations entre étiquettes et expressions par un graphe spatialisé afin de rapprocher les mots souvent utilisés ensemble. L'objective de la méthode est simple : il s'agit d'identifier des univers de discours liés aux différentes façons de désigner les personnes se déplaçant pour des raisons environnementales.

Le lecteur nous pardonnera d'aborder ici des détails techniques et quelque peu fastidieux : il n'y a pas d'autre moyen d'exposer la validité de notre démarche.

Le point de départ est l'interrogation du moteur de recherche Google.com (dans la version la plus «standard » possible ${ }^{26}$ ) avec les requêtes présentées dans le tableau suivant :

24. Lazer D., Pentland A., Adamic L., Aral S., Barabasi A.-L., Brewer D., Christakis N., et al., "Computational social science", Science, 323, 5915, 2009, pp.721-3.

25. Venturini T., «Great Expectations : méthodes quali-quantitative et analyse des réseaux sociaux », in Fourmentraux J.P. (ed.), L’Ère Post-Média, Paris, Hermann, 2012.

26. Il est connu que le moteur de recherche Google.com “personnalise” les résultats des recherches qui lui adressés en se fondant sur l'historique de recherche et de navigation, le profil Google de l'utilisateur, le lieu d'où provient la requête, la langue du navigateur et d'autres variables qui ne sont pas complètement connues. Par conséquent, deux utilisateurs posant à Google la même requête peuvent très bien obtenir des réponses différentes. Nous avons essayé de fournir à Google le moins d'informations possibles sur notre identité afin d'obtenir les résultats les moins personnalisés possible. Mais en toute occurrence, étant donné que la personnalisation concerne surtout l'ordre des résultats et que nous avons visité les 100 premiers résultats pour chaque requête, nous sommes confiants quant au fait que la personnalisation de Google ne devrait pas avoir trop biaisé les résultats obtenus. 


\begin{tabular}{|l|l|}
\hline Requête & Nombre de pages moissonnées \\
\hline "Climate refugee" & 84 pages \\
\hline "Environmental migrant" & 76 pages \\
\hline "Climate migrant" & 69 pages \\
\hline "Environmental Refugee" & 72 pages \\
\hline "Eco-migrant" & 80 pages \\
\hline "Ecological migrant" & 64 pages \\
\hline "Ecological refugee" & 65 pages \\
\hline "Climate-induced migrant" & 12 pages \\
\hline "Climate-induced refugee" & 16 pages \\
\hline "Environmentally-induced migrant" & 62 pages \\
\hline "Environmental displacee" & 86 pages \\
\hline "Environmentally displaced person" & 48 pages \\
\hline "Eco-refugee" & 79 pages \\
\hline "Ecologically displaced person" & 90 pages \\
\hline
\end{tabular}

Tableau 1. Résultats considérés pour chaque requête

Pour chacune des requêtes nous avons visité les 100 premiers résultats (ou moins, si la requête en produisait moins) et, quand il s'agissait de pages web, nous avons moissonné leur texte ${ }^{27}$. Le corpus ainsi constitué est composé du texte de 903 pages. La constitution du corpus a été réalisée le 9 février 2012. Il est important d'indiquer la date, car notre analyse reflète la situation du débat à cette date. Contrairement à ce qu'on croit souvent, le web a très peu de mémoire. Il est donc très difficile de réaliser des analyses diachroniques du débat sur le Web à moins de disposer d'un corpus de pages web archivé dans le temps ${ }^{28}$.

Nous avons ensuite employé le moteur d'extraction sémantique Alchemy ${ }^{29}$ pour extraire les ngrams et les entités nommées présents dans chacun des 903 textes de notre corpus. Par ngram, on entend une expression de $\mathrm{n}$ mots apparaissant plusieurs fois dans le même texte (par exemple anthropogenic climate change). Par entités nommées, on entend un ngram qui est reconnu par Alchemy comme faisant référence à une personne (par exemple Richard

27. Pour des raisons techniques, nous n’avons pas pu récupérer le texte correspondant aux résultats qui n’étaient pas des pages html (vidéos, animations flash, documents PDF, présentations PowerPoint, documents DOC...). Ceci explique pourquoi nous n’avons pu récupérer la totalité des 100 premiers résultats pour aucune des requêtes.

28. Le médialab de Sciences Po travaille à la réalisation d'un outil capable de permettre cet archivage, mais à ce jour cet type d'analyse n'est pas encore possible.

29. http://www.alchemyapi.com/ 
Black), à une organisation (par exemple l'OIM) ou à un lieu géographique (par exemple Tuvalu). Par la suite, nous désignerons ngrams et entités nommées par le terme générique d' « expressions ».

Une précaution méthodologique s'impose. Tout à l'heure, nous avons affirmé que l'objectif de notre analyse était de dégager les « univers des discours » autour des différentes étiquettes des migrations liées à l'environnement. Le choix de nous concentrer sur les expressions extraites des pages web comporte cependant la réserve que l'analyse que nous avons menée n'est pas une analyse de discours au sens strict du terme. Une analyse de ce type aurait demandé une lecture méticuleuse, difficile à réaliser pour un corpus aussi vaste que celui que nous avons collecté. Afin de garder une large couverture des débats en ligne, nous avons donc dû nous contenter d'une analyse plus légère et nous limiter à compter l'occurrence d'expressions lexicales dans les pages de notre corpus. Nous sommes bien conscients que la façon d'utiliser une certaine expression, le contexte rhétorique et pragmatique sont souvent plus importants que le contenu de l'expression elle-même.

Pour contrebalancer cette difficulté, deux remèdes ont été mis en place. En premier lieu, l'interprétation des résultats de notre analyse, loin de s'appuyer exclusivement sur les cartes que nous avons produites, se fonde également sur une connaissance experte du débat. Deuxièmement, nous avons essayé de récolter et d'analyser un très grand nombre d'expressions afin de reconstruire en partie le contexte d'usage.

Le traitement par Alchemy des 903 textes du corpus a restitué plus de 50000 expressions 30 (52 542 exactement) : un nombre d'expressions beaucoup trop grand pour pouvoir les analyser et les interpréter. Un travail d'analyse préliminaire a donc été nécessaire pour réduire les expressions à une quantité plus raisonnable. Pour cela, nous avons d'abord appliqué un premier tri automatique en éliminant toutes les expressions les moins fréquentes. En particulier, nous avons éliminé toutes les expressions apparaissant dans moins de trois des textes du corpus et toutes les expressions moins fréquentes. Le tri automatique a permis d'exclure 90,5 \% des expressions trouvées par Alchemy, en réduisant le nombre d'expressions à 4995 . Nous avons ensuite trié manuellement les expressions restantes, en éliminant les expressions trop génériques et ambiguës (par exemple major issue, ou significant consequences) et en fusionnant les synonymes (par exemple United Kingdom et $U K$ ). Cela nous a permis d'obtenir un corpus de 609 expressions. Nous avons ensuite manuelle-

30. Dans le paramétrage de l'analyse de Alchemy, nous avons choisi de n'extraire que les n-grams de langue anglaise. Malgré l'évident intérêt que cela aurait eu, une analyse multilingue aurait posé l'énorme problème de l'équivalence des expressions dans les différentes langues. On sait bien, par exemple, que «développement durable » et «sustainable development » ne désignent pas tout à fait la même chose. C’est pourquoi il nous a semblé plus prudent de nous limiter à une seule langue : nous avons choisi l'anglais comme langue du débat international. 
ment corrigé la classification des entités nommées de Alchemy, en identifiant tous les acteurs (individuels ou collectifs) et les lieux (continents, pays, régions, villes...) présents dans notre liste finale d'expressions.

Nous avons ensuite exporté un réseau non-pondéré de documents et d'expressions (voir Fig. 1). Les nœuds de ce réseau sont les 903 textes et les 609 expressions que nous avons retenus dans notre corpus final. Le réseau est biparti, car ses arcs ne connectent jamais deux nœuds du même type (deux textes ou deux expressions), mais toujours des nœuds de type différent : chaque expression est connectée à tous les textes où elle apparaît, et chaque texte est connecté à toutes les expressions qu'il contient.

Toutes les phases décrites jusqu'ici ont été réalisées à l'aide de ANTA, un logiciel développé par le médialab de Sciences Po ${ }^{31}$.Toutes les phases suivantes et impliquant l'analyse du réseau des documents et entités ont été réalisées à l'aide de GEPHI (gephi.org), un logiciel d'analyse de réseaux co-développé par le médialab de Sciences Po ${ }^{32}$.

Le choix d'une analyse et d'une représentation par les réseaux mérite d'être brièvement discuté, d'autant plus que les données analysées ne sont pas de nature intrinsèquement relationnelle. Pour réaliser nos cartes, nous avons en effet choisi d'ignorer les liens explicites présents sur les pages web constituant notre corpus, pour nous concentrer sur les liens indirects de co-occurrence d'expressions. Cette information aurait pu très bien se prêter à une analyse matricielle : les même données pourraient être présentées dans un tableau ayant en colonnes les documents (ou les requêtes) et en lignes les expressions sélectionnées. Ce tableau aurait pu être analysé avec des méthodes statistiques et visualisé par des diagrammes classiques (histogrammes, camemberts, etc.). Si nous avons décidé d'utiliser une analyse de réseaux, c'est parce que ce type d'analyse nous semble particulièrement adapté aux objectifs d'exploration de cet article. S'agissant de la première tentative d'analyse à large échelle du débat en ligne sur les migrations environnementales, notre analyse ne peut être qu'exploratoire ${ }^{33}$. Dans cet article, nous n'avons pas des hypothèses précises à tester. Notre objectif est plutôt de défricher les grandes tendances du débat en ligne, en préparant le terrain pour des études plus approfondies. Grâce à leur capacité à faciliter à la fois l'observation des données individuelles (chaque nœud dans le réseau reste visible) et des caractéristiques globales (densité, clustering, centralité...), les réseaux nous semblent l'outil idéal pour ce travail d'exploration ${ }^{34}$, en particulier s'ils sont spatialisés avec un algorithme force-vecteur.

31. Voir Venturini T., Guido D., "Once Upon a Text: An ANT Tale in Text Analysis", Sociologica, à paraître, 2012.

32. Les deux outils sont diffusés avec une licence open-source et leur code est disponible ici : http://github.com/medialab.

33. Tukey J. W., Exploratory Data Analysis, Reading, Addison-Wesley, 1977. 


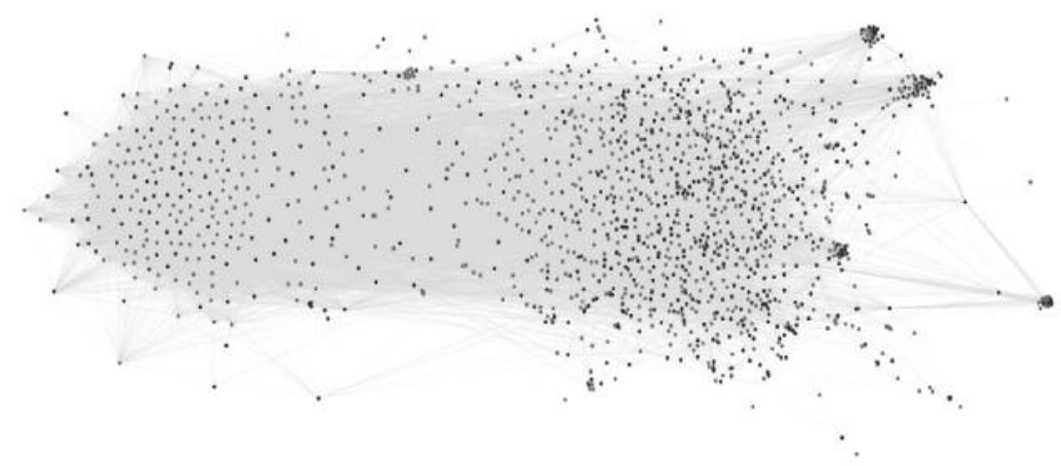

Figure 1. Le réseau biparti des expressions (en gris foncé) et des documents (en gris clair)

Le réseau de la Figure 1, ainsi que les réseaux suivants, sont spatialisés à l'aide d'un algorithme force-vecteur (en l'occurrence ForceAtlas2 LinLog mode ${ }^{35}$ ). Cette étape est très importante : elle consiste à positionner les nœuds du graphe en utilisant un algorithme qui simule un système de forces physiques. Dans ce système, les nœuds exercent une force de répulsion les uns sur les autres, tandis que les arcs fonctionnent comme des élastiques, gardant proches les nœuds qu'ils connectent. Une fois que l'algorithme trouve sa position d'équilibre, la position relative des nœuds devient significative. Si, par exemple, deux documents se retrouvent proches dans le graphe, c'est parce qu'ils partagent plusieurs expressions. Ou encore, si une expression est liée à deux documents seulement, elle va occuper une position intermédiaire entre les deux.

Le réseau présenté en Figure 1 représente les «données brutes » de notre analyse, mais il est beaucoup trop complexe pour pouvoir être interprété. Afin de rendre le réseau lisible, nous lui avons fait subir plusieurs transformations graphiques et mathématiques. En premier lieu, nous avons décidé d'éliminer les documents liés aux requêtes Climate-induced migrant (12 documents) et Climate-induced refugee (16 documents). Ayant généré beaucoup moins de documents que les autres, leur analyse risquait en effet d'être biaisée par l'insuffisance de l'échantillon de textes considérés. Nous avons ensuite groupé les 875 documents dans 12 groupes correspondant aux requêtes qui avaient permis de les trouver. Une conséquence importante de cette action de regroupement est la pondération des liens du graphe ${ }^{36}$. Le résultat est visible en Figure 2.

34. Venturini T., «Great Expectations : méthodes quali-quantitative et analyse des réseaux sociaux ", op.cit.

35. Jacomy M., Heymann S., Venturini T. et Bastian M., ForceAtlas2, a graph layout algorithm for handy network visualization, 2011 : http://www.medialab.sciences-po.fr/fr/publicationsfr/, consulté le 20 décembre 2012.

36. Le pois d'un arc connectant une expression et une requête est égal au nombre de résultats de la requête contenant cette expression. 


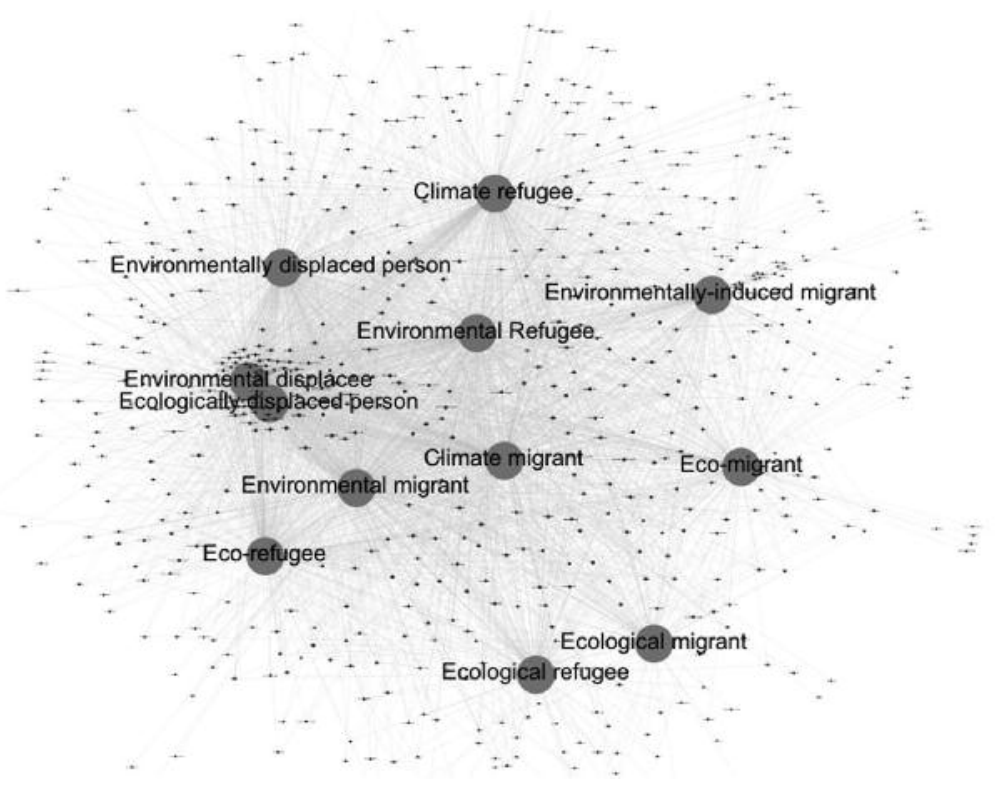

Figure 2. Le réseau des expressions et des requêtes

Tout en étant beaucoup plus lisible que la figure précédente (il est maintenant possible de lire les noms des requêtes, et donc de voir quelles requêtes sont proches parce qu'elles partagent le même contexte lexical), la Figure 2 reste encore difficile à interpréter. En particulier, le nombre d'expressions est encore trop grand pour qu'on puisse les afficher dans le graphe.

Afin de réduire le nombre d'expressions sur la carte, nous avons suivi deux stratégies. En premier lieu, nous avons enlevé toutes les expressions connectées à une seule des requêtes ou à l'ensemble des douze requêtes. Ces entités, bien sûr, ne sont pas moins importantes que les autres, mais pour elles une visualisation par liste (comme celle présentée dans le Tableau 2 ci-contre) peut remplacer une visualisation par graphe.

Nous avons ainsi enlevé 76 expressions, mais les 533 expressions restantes sont encore trop nombreuses pour pouvoir être lues dans le graphe. Il convient donc de scinder le graphe en trois sous-graphes, en distinguant les 76 expressions faisant référence à des noms d'acteurs individuels ou collectifs (Fig. 3) et les 100 toponymes géographiques (Fig. 4). Dans les deux images suivantes, la taille des nouds-expressions est proportionnelle au nombre de documents (pages web) dans lesquels ils apparaissent. Quant à la couleur des nœuds, elle dépend du nombre de requêtes auxquelles ils sont connectés : plus ce nombre est grand, plus la couleur du nœud est foncée. 


\begin{tabular}{|c|c|c|c|c|c|}
\hline Label & $\begin{array}{l}\mathrm{N}- \\
\text { docs }\end{array}$ & Requête & Label & \begin{tabular}{|l}
$\mathrm{N}-$ \\
docs
\end{tabular} & Requête \\
\hline asia & 126 & Toutes & climate refugee hoax & 5 & Climate refugee \\
\hline pakistan & 67 & Toutes & human cost & 4 & Climate refugee \\
\hline social networks & 23 & Toutes & political migrants & 3 & Environmental migrant \\
\hline environmentalists & 27 & Toutes & recent earthquake & 3 & Environmental migrant \\
\hline european commission & 292 & Toutes & nansen passport & 4 & Environmental migrant \\
\hline unhcr & 227 & Toutes & migration control & 3 & Climate migrant \\
\hline security & 79 & Toutes & climate shifts & 2 & Climate migrant \\
\hline water & 171 & Toutes & climate debate & 2 & Climate migrant \\
\hline australia & 249 & Toutes & climate stresses & 5 & Climate migrant \\
\hline bangladesh & 296 & Toutes & early eco migrant & 4 & Eco-migrant \\
\hline urgency & 99 & Toutes & human smugglers & 23 & Eco-migrant \\
\hline urban areas & 37 & Toutes & northern hemisphere barren & 3 & Eco-migrant \\
\hline biofuels & 63 & Toutes & undocumented immigrants & 3 & Eco-migrant \\
\hline desertification & 154 & Toutes & logical correlation & 3 & Eco-migrant \\
\hline human rights & 106 & Toutes & public housing & 3 & Eco-migrant \\
\hline emergent crisis & 251 & Toutes & labour rights & 2 & Ecological migrant \\
\hline $\begin{array}{l}\text { geneva refugee con- } \\
\text { vention }\end{array}$ & 96 & Toutes & new houses & 8 & Ecological migrant \\
\hline $\begin{array}{l}\text { international frame- } \\
\text { work }\end{array}$ & 119 & Toutes & environmental rights & 3 & Ecological refugee \\
\hline natural disaster & 225 & Toutes & our common future & 4 & Ecological refugee \\
\hline dry land & 113 & Toutes & southern dust bowl & 3 & Ecological refugee \\
\hline indigenous people & 81 & Toutes & cara nine & 10 & Ecological refugee \\
\hline unep & 482 & Toutes & philosophical traditions & 3 & Ecological refugee \\
\hline sea level rise & 371 & Toutes & marc sheppard & 22 & $\begin{array}{l}\text { Environmentally-induced } \\
\text { migrant }\end{array}$ \\
\hline legal & 276 & Toutes & shakir husain & 14 & $\begin{array}{l}\text { Environmentally-induced } \\
\text { migrant }\end{array}$ \\
\hline environmental factors & 104 & Toutes & whacky climate refugees & 22 & $\begin{array}{l}\text { Environmentally-induced } \\
\text { migrant }\end{array}$ \\
\hline $\begin{array}{l}\text { environmental degra- } \\
\text { dation }\end{array}$ & 291 & Toutes & terrifying words & 20 & $\begin{array}{l}\text { Environmentally-induced } \\
\text { migrant }\end{array}$ \\
\hline climate change & 522 & Toutes & nobel laureate & 20 & $\begin{array}{l}\text { Environmentally-induced } \\
\text { migrant }\end{array}$ \\
\hline pacific ocean & 48 & Toutes & environmental horror & 14 & $\begin{array}{l}\text { Environmentally-induced } \\
\text { migrant }\end{array}$ \\
\hline new scientist & 70 & Toutes & barbara boxer & 14 & $\begin{array}{l}\text { Environmentally-induced } \\
\text { migrant }\end{array}$ \\
\hline india & 296 & Toutes & $\begin{array}{l}\text { impossible doomsday sce- } \\
\text { narios }\end{array}$ & 14 & $\begin{array}{l}\text { Environmentally-induced } \\
\text { migrant }\end{array}$ \\
\hline south asia & 41 & Toutes & excessive statements & 14 & $\begin{array}{l}\text { Environmentally-induced } \\
\text { migrant }\end{array}$ \\
\hline
\end{tabular}




\begin{tabular}{|c|c|c|c|c|c|}
\hline Label & $\begin{array}{l}\mathrm{N}- \\
\text { docs }\end{array}$ & Requête & Label & $\begin{array}{l}\mathrm{N}- \\
\text { docs }\end{array}$ & Requête \\
\hline & & & robust findings & 12 & Environmentally-induced migrant \\
\hline & & & rajendra k. pachauri & 12 & Environmentally-induced migrant \\
\hline & & & eua & 10 & Environmentally-induced migrant \\
\hline & & & pat carlson & 12 & Environmentally-induced migrant \\
\hline & & & corrupt governments & 12 & Environmentally-induced migrant \\
\hline & & & environmental groups & 4 & Environmentally-induced migrant \\
\hline & & & robust science & 10 & Environmentally-induced migrant \\
\hline & & & lisa jackson & 12 & Environmentally-induced migrant \\
\hline & & & new country & 8 & Environmentally-induced migrant \\
\hline & & & operative phrase & 4 & Environmentally-induced migrant \\
\hline & & & apocalyptic view & 4 & Environmentally-induced migrant \\
\hline & & & intense heat waves & 12 & Eco-refugee \\
\hline & & & rural vietnam & 9 & Eco-refugee \\
\hline & & & territorial rights & 12 & Ecological refugee \\
\hline
\end{tabular}

Tableau 2 (cette page et la précédente).

Expressions connectées à l'ensemble des 12 requêtes ou à une seule d'entre elles

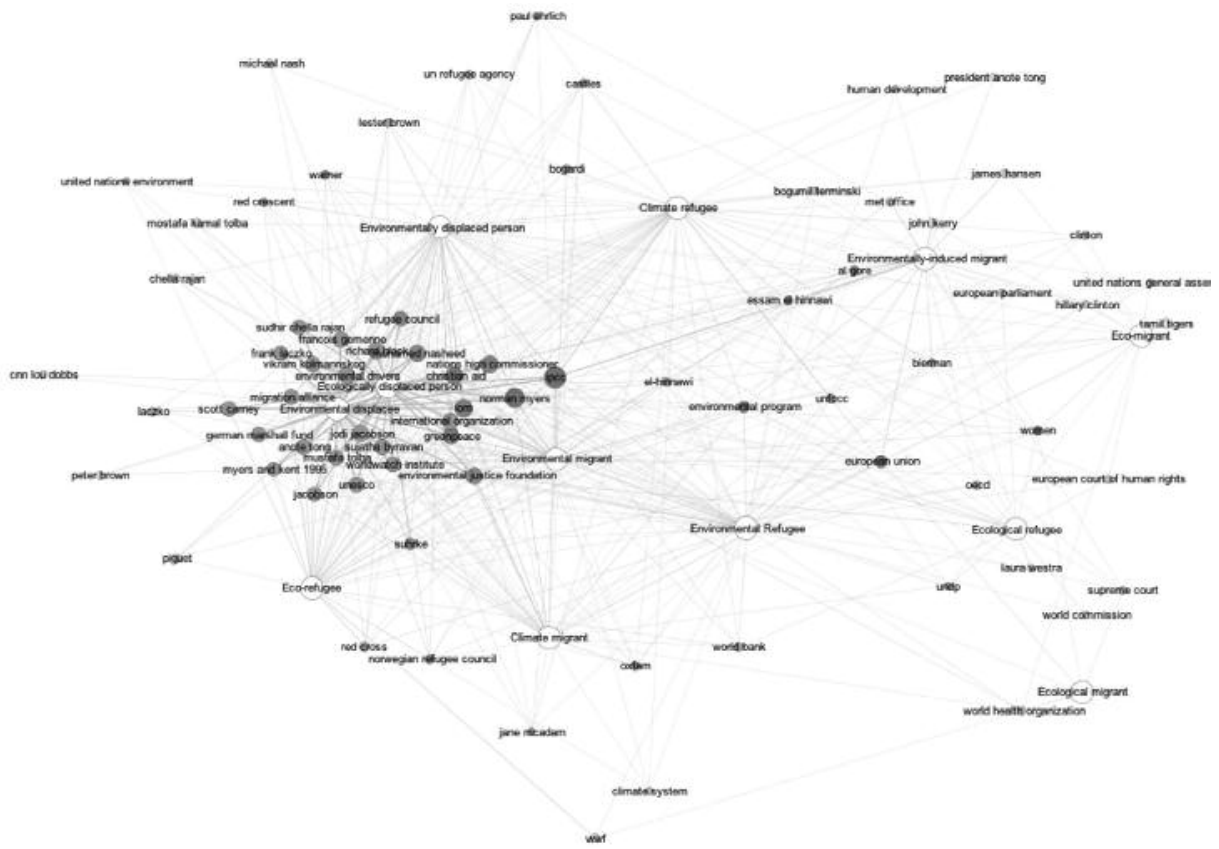

Figure 3. Le réseau des noms d'acteurs (gris) et des requêtes (blanc) 


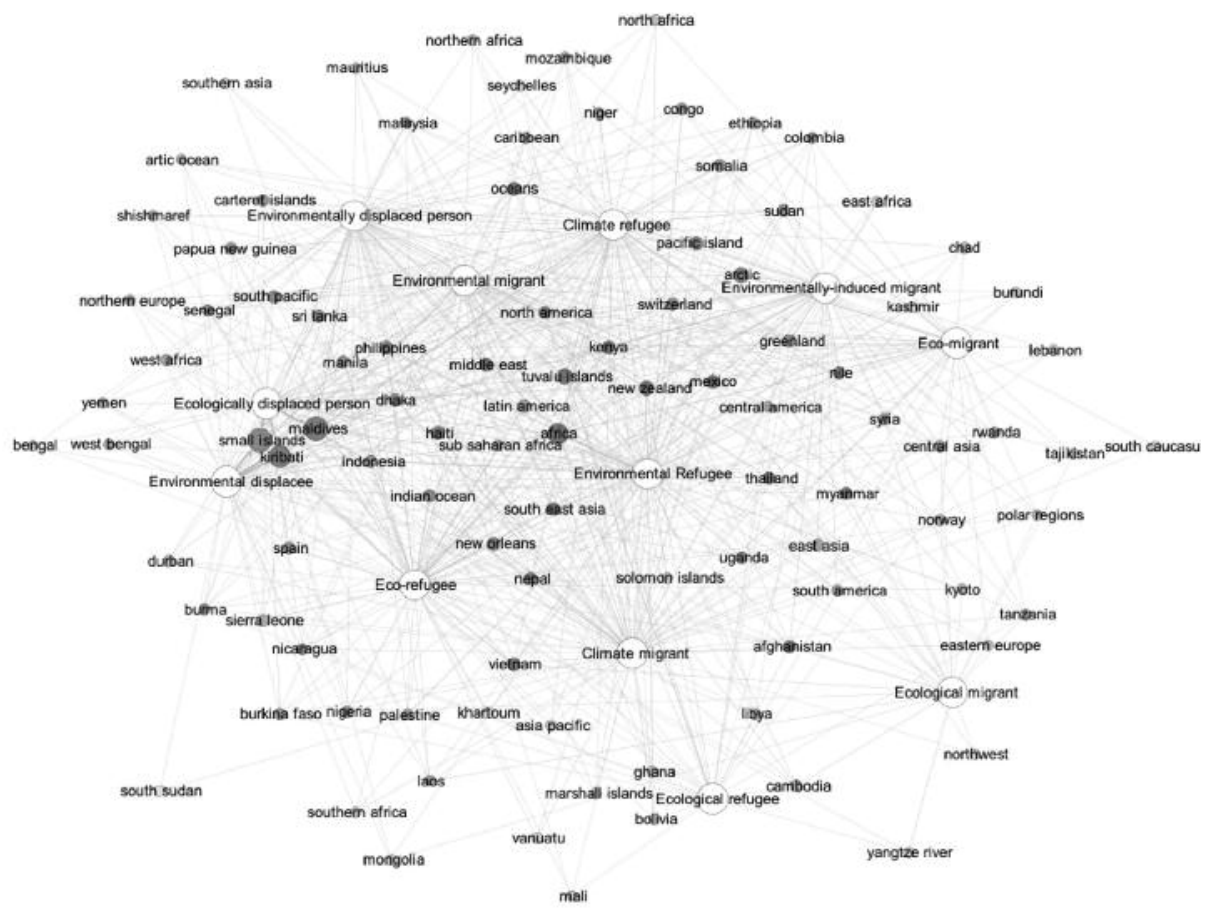

Figure 4. Le réseau des toponymes (gris) et des requêtes (blanc)

Une fois enlevés les noms d'acteurs et les toponymes, il est possible de dessiner un réseau - enfin lisible - des 357 expressions restantes (Fig. 5, page suivante). Il est intéressant de remarquer que, dans les Figures 3, 4 et 5, la position relative des nouds représentant les requêtes ne change pas, ce qui en prouve la robustesse.

\section{Discussion des cartes}

Ce qui frappe au premier abord, à la lecture des cartes, c'est avant tout l'extraordinaire connexion qui existe entre les différents termes utilisés et les personnes, lieux, organisations et concepts auxquels ils sont associés. Ce que les cartes font apparaître en premier lieu, c'est combien chaque terme est associé à une multitude d'autres acteurs et concepts. Les expressions associées à une seule des étiquettes employées comme requêtes ne sont qu'au nombre de 45 sur les 600 que nous avons retenues pour l'analyse. Quatre des douze étiquettes ('environmental refugee', 'environmental displacee', 'environmentally displaced person', 'ecologically displaced person') ne sont connectées exclusivement à aucun acteur, lieu ou concept, tandis que quatre autres ('climate refugee', 'environmental migrant', 'eco-refugee', 'ecological refugee’) n'ont plus que trois expressions qui leur sont propres (voir le Tableau 2). 
Figure 5. Le réseau des expressions (gris) et des requêtes (blanc)

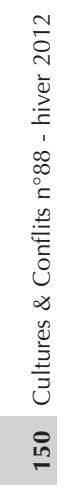

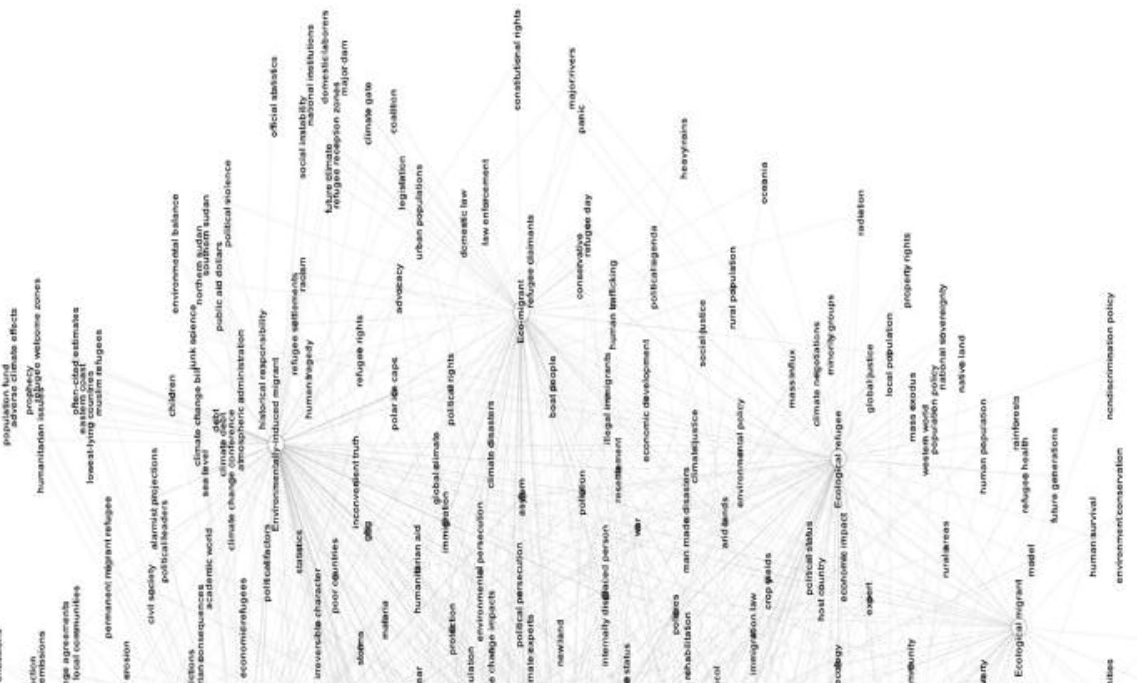

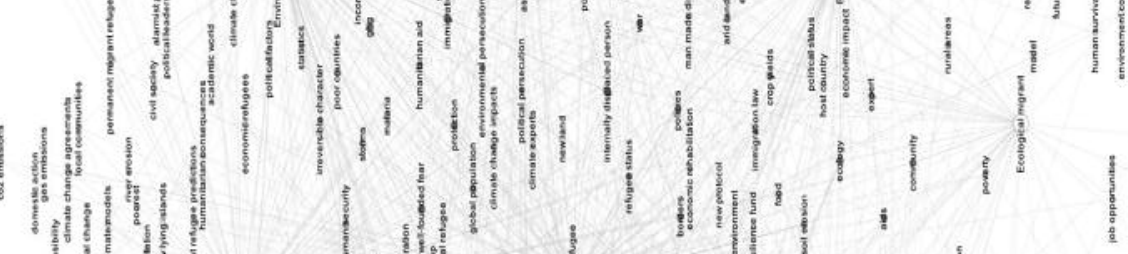<smiles>C#CC1CCC1C#N</smiles>
(4)

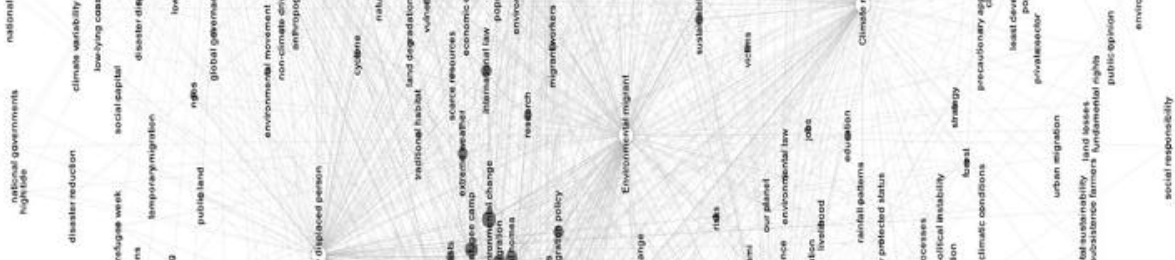

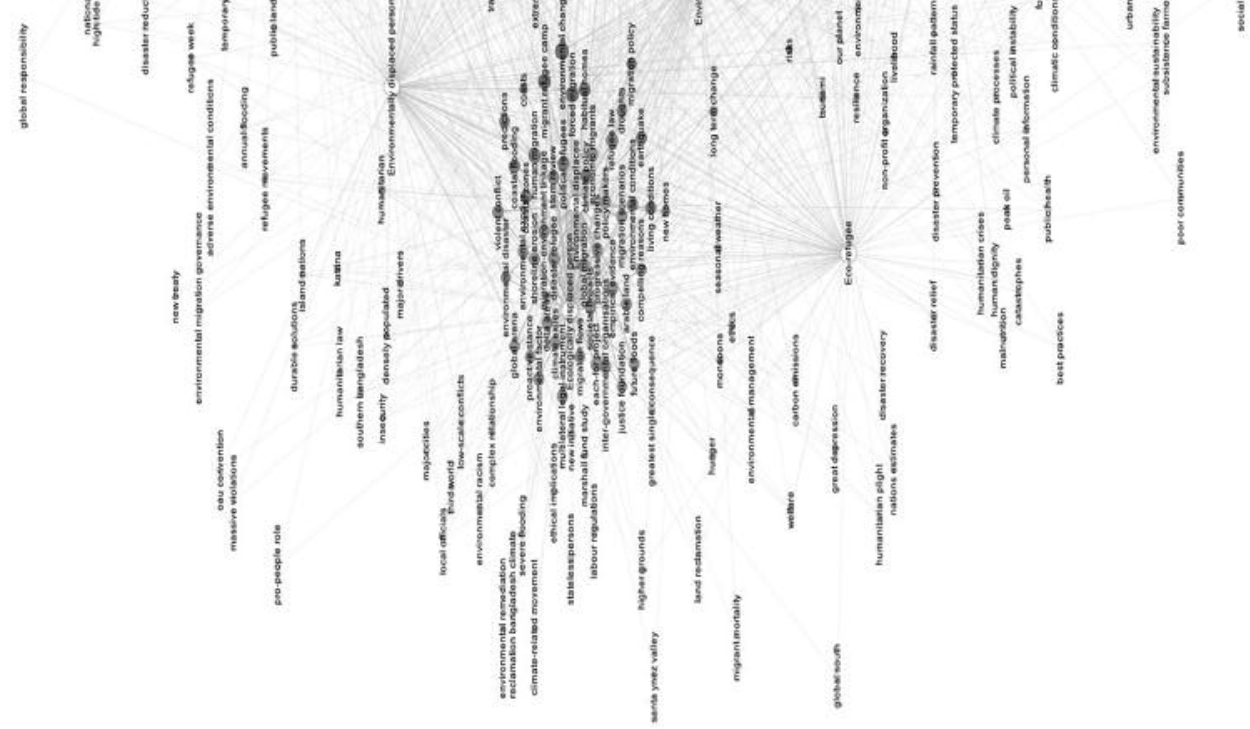


Tout se passe comme si les différents termes étaient utilisés relativement indistinctement les uns des autres. Le fait que des expressions comme 'environmental degradation', 'water', 'desertification', 'dry land', 'natural disaster', 'sea-level rise', et même 'climate change' apparaissent dans les résultats de toutes les requêtes (voir le Tableau 2) montre bien l'existence d'un consensus sur les principales causes de ces type de migration. On peut faire le même constat pour les acteurs-clés ('UNHCR', 'indigenous people', 'European Commission', 'Geneva refugee convention'), pour les enjeux liés au phénomène ('security', 'urgency', 'human rights', 'emergent crisis', 'international framework', 'legal') et les régions prioritairement concernées ('Asia', 'Pakistan', 'Australia', 'Bangladesh', 'Pacific Ocean', 'India', 'South Asia').

Cette situation reflète bien le vide catégoriel dans lequel se trouvent aujourd'hui les migrations liées à l'environnement. En soi, la multitude de termes n'est pas un véritable problème. Le problème tient davantage au fait qu'elle désigne manifestement une même réalité : chaque terme est associé aux mêmes concepts, et largement utilisé par les mêmes acteurs. On trouve là, au fond, tout le problème du vide catégoriel : si tout exercice de catégorisation est délicat, l'absence de catégories, de typologie, l'est sans doute tout autant. L'absence d'une typologie clairement établie handicape considérablement la recherche d'une définition, et, partant, la possibilité d'établir des estimations chiffrées et de développer des politiques publiques adéquates ${ }^{37}$.

Un deuxième élément marquant est que le terme le plus générique, 'migrant environnemental' (en anglais 'environmental migrant'), n'apparaît pas plus central que d'autres. De même, les termes qui contiennent le mot " réfugié ", pourtant maintes fois réfuté par les chercheurs, n'apparaissent pas plus périphériques que les autres. Il convient de rappeler ici que la position des nœuds est significative, puisque nous avons spatialisé nos réseaux à l'aide d'un algorithme de force-vecteur. En particulier, la centralité dans la carte indique la connexion avec une terminologie neutre ou consensuelle (c'est-à-dire connectée à la plupart des étiquettes). Ceci tendrait à montrer que les efforts de nombreux chercheurs et organisations internationales pour imposer le vocable de « migrant » plutôt que celui de « réfugié » (en anglais « refugee ») n'ont guère produit d'effet ${ }^{38}$. L'un comme l'autre semblent occuper une position périphérique et être donc incapables de constituer un terrain de rencontre pour les différents discours sur climat et migration.

Entre « migrant » et « réfugié », c'est finalement un troisième terme qui semble s'imposer. Que l'on considère la carte des lieux, des acteurs ou des

37. Dun O., Gemenne F., “Defining 'environmental migration’”, Forced Migration Review, 31, 2008, pp.10-11.

38. Voir par exemple une note de l'Organisation Internationale pour les Migrations sur ce sujet : International Organization for Migration, "Discussion note: Migration and the Environment." in MC/INF/288, vol. MC/INF/288, Genève, IOM, 2007. 
concepts, ce sont à chaque fois les termes qui comprennent le mot « déplacé » (en anglais « displaced») qui occupent le cœur de la carte. De façon plus significative encore, dans toutes les cartes, les expressions 'environmental displacee’ et 'ecologically displaced person' sont connectées à un groupe très dense d'expressions partagées par un grand nombre de pages web, et connectées à la plupart des étiquettes (comme indiqué respectivement par leur taille plus grande et leur couleur plus foncée). Ce groupe contient les expressions moins connotées, dont la neutralité finit par faire consensus. Le fait que 'environmental displacee' et 'ecologically displaced person' soient très fortement connectés à ces expressions indique que ces deux étiquettes sont la marque d'un discours qui évite les termes plus controversés (situés plutôt dans la partie droite des cartes).

Quoiqu'il puisse parfois apparaître comme excessivement clinique ${ }^{39}$, le terme de «déplacé » possède l'avantage de signifier une réalité proche de celle portée par le terme « réfugié »-c'est-à-dire celle d'une migration forcée - sans être strictement défini par une convention internationale, qui rendrait son usage juridiquement incorrect. En d'autres termes, « déplacé » permet de dire sensiblement la même chose que "réfugié » sans être astreint aux mêmes restrictions que celles imposées par la Convention de Genève de 1951, qui définit le statut de réfugié. Quoique les juristes aient beaucoup insisté sur l'abus de langage que constituait l'usage du terme « réfugié " dans ce type de situation, le terme est pourtant d'usage courant, et fait référence à toute personne cherchant un refuge, indépendamment des conditions définies par la Convention. "Réfugié » a donc bien une double signification : l'une est restrictive et juridique ; l'autre est beaucoup plus large et d'usage commun. S'il existe des raisons pour refuser l'usage du terme « réfugié » sur le plan juridique, il en existe d'autres, peut-être plus importantes, sur le plan sociologique. Et la première de ces raisons est sans doute que le terme est généralement refusé par ceux qu'on appelle « réfugiés ", ainsi que par les États qui sont supposés les accueillir, voire même parfois par les États d'origine, qui craindraient d'être assimilés à des États qui persécutent leurs populations.

Passons à présent à l'examen individuel des différentes cartes. La carte qui associe les différents termes avec les acteurs (Fig. 3) est avant tout caractérisée par la grande concentration d'acteurs autour des termes qui intègrent le mot « déplacé », soit " déplacé environnemental » (en anglais 'environmental displacee'), «personne déplacée pour des raisons écologiques » (en anglais 'ecologically displaced person’) ou «personne déplacée pour des raisons environnementales " (en anglais 'environmentally displaced person'). Ces termes, compromis entre "réfugiés » et «migrants ", rassemblent un petit groupe d'acteurs incontournables et cités par un grand nombre de pages web connectées aux différentes étiquettes. Par exemple, on trouve dans ce groupe

39. Voir par exemple la citation de Lou Dobbs reproduite dans l'introduction. 
l'Organisation Internationale pour les Migrations, le Haut Commissariat des Nations Unies pour les Réfugiés, le chercheur Richard Black ou l'ancien président des Maldives Mohamed Nasheed. Même si plusieurs travaux de recherche ont mis en évidence les nombreuses différences qui existaient entre les discours des différentes organisations et l'évolution de ces discours au fil de temps ${ }^{40}$, ces différences ne semblent pas se répercuter dans les expressions utilisées dans le débat en ligne. Aucune institution ou organisation n'est connectée à une seule étiquette. Parmi les acteurs collectifs, seul le WWF se distingue par une association privilégiée avec les termes de "réfugié » (tout en étant aussi lié à 'environnemental migrant'). Parmi les acteurs individuels, on rencontre plus des connexions exclusives : la Sénatrice américaine Barbara Boxer, par exemple, ou le Président du GIEC Rajendra K. Pachauri ne sont liés qu'à l'étiquette 'environmentally-induced migrants' (voir Tableau 2).

La carte des différents lieux (Fig. 4), quant à elle, fait apparaître une autre image. La concentration autour des termes " déplacés » apparaît moins marquée qu'ailleurs, et les noms de lieux sont plus uniformément répartis autour des différents termes. On notera avec intérêt que la ville de La NouvelleOrléans reste étroitement associée aux termes d' " éco-réfugié » ou de "réfugié écologique ». Les petits États insulaires restent plutôt associés aux termes de "déplacés ", tandis que les zones affectées par des sécheresses sont plus proches des termes de «migrants». Autour des "déplacés environnementaux » et termes apparentés, on trouve ici les " petites îles », les Maldives, Kiribati, le Pacifique Sud, Tuvalu ou la Papouasie Nouvelle-Guinée. Ceci s'explique sans doute par le fait que les mouvements de populations liés à la hausse du niveau des mers sont généralement associés à des déplacements forcés, en particulier dans la perspective de la possible disparition future de territoires. À l'inverse, plusieurs travaux ont mis en évidence que la mobilité était fréquemment une stratégie utilisée par les populations pour s'adapter aux sécheresses ${ }^{41}$. Rien d'étonnant donc à ce que les pays et régions affectés par des sécheresses - Niger, Ethiopie, Soudan, Tchad, Afrique de l'Est - soient plus volontiers associés au terme de «migrant », qui laisse supposer une mobilité choisie plutôt que subie.

Enfin, dans la carte des concepts associés aux différents termes (Fig. 5), il est intéressant de se concentrer d'abord sur le groupe de concepts situés autour des termes de "déplacés ». Ce groupe d'expressions relativement «consensuelles» (puisque présentes dans de nombreux documents et connectées à la plupart des étiquettes) semble être constitué par deux types de termes. D’une part, on y trouve des termes neutres qui semblent faire consensus pour leur manque de connotation (par exemple empirical evidence, living condi-

40. Ollitrault S., «De la sauvegarde de la planète à celle des réfugiés climatiques : l'activisme des ONG », Revue Tiers-Monde, 204, 2010, pp. 19-34.

41. Van Der Geest K. "North-South migration in Ghana: What role for the environment?", International Migration, 49, 1, 2011, pp. 69-94. 
tions, new homes, compelling reasons, environmental factor, migration-environment linkage, predictions, global arena, policy makers, societal impacts...). D'autre part, on y trouve aussi des mots bien plus connotés, mais apparemment incontournables dans le débat (par exemple refugee law, forced migration, migration policy, climate exiles, justice foundation, migrant refugee camp, environmental exodus). Comme souvent dans les controverses se prolongeant sur plusieurs années sans trouver une solution univoque, le désaccord sur le lien entre migration et environnement tend à se structurer. Les acteurs commencent à partager non seulement les bases de la discussion, mais aussi les questions qui les divisent.

Dans le débat sur le lien entre environnement et migrations, toutefois, l'accord sur le désaccord est loin d'être parfait. On peut voir ceci dans la structure polarisée de la carte des enjeux. Si le groupe consensuel que nous avons identifié autour des étiquettes 'environmental displacee' et 'ecologically displaced person' représente le cœur de la carte, il n'en occupe pas le centre, qui semble occupé par une sorte de "vide structurel » ${ }^{42}$. Au cœur des déplacés s'oppose, à droite dans la carte, une nébuleuse d'expressions liées aux étiquettes 'climate refugee', 'environmentally-induced migrant', 'eco-migrant', 'ecological refugee', 'environmental refugee', 'ecological migrant'. Cette nébuleuse est constituée par des expressions marquées politiquement et moralement (par exemple asylum, boat people, man made disaster, climate disaster, buman tragedy, racism, political violence, climate justice, social justice, refugee bealth, nondiscrimination policy...), qui par conséquent tendent à apparaître dans moins de pages web (d'où leur taille plus petite) et à être liées à moins d'étiquettes (d'où leur couleur plus claire). Ce sont ces expressions qui mesurent l'extension du débat sur le lien entre environnement et migrations. Ce sont ces expressions qui créent la controverse.

\section{Conclusion}

Si plusieurs phénomènes récents, comme l'ouragan Katrina et les tremblements de terre à Haïti, ont imposé de manière indiscutable la reconnaissance du lien entre environnement et migrations, l'interprétation de ce lien n'est pas pour autant évidente. Tant dans les arènes scientifiques que dans les organisations internationales, le débat concernant la définition des millions des personnes touchées par des dégradations de leur environnement reste profondément ouvert. Plusieurs termes ont été proposés pour les définir : refugié (climatique, écologique ou environnemental), migrant (climatique, écologique ou environnemental) ou personne déplacée (en raison de l'environnement ou du climat). Malgré les efforts des chercheurs pour définir le sens et les effets de chacun de ces mots (et notamment sur les conséquences politiques du terme

42. Burt R. S., Structural Holes: The Social Structure of Competition, Cambridge (MS), Harvard University Press, 1995. 
« réfugié » à la place du terme « migrant » ou « déplacé »), ces mots ont occupé la discussion publique de manière chaotique et souvent controversée.

Dans cet article, nous avons essayé d'éclaircir cette controverse en étudiant les usages de ces mots dans le débat public sur Internet. Pour ce faire, nous avons employé une nouvelle méthode numérique qui nous a permis, à travers l'interrogation du moteur de recherche Google.com, de collecter les pages web où la discussion autour des différentes définitions des migrants climatiques était la plus visible. Grâce à une analyse des expressions contenues dans ces pages, nous avons obtenu des cartes sémantiques qui ont nous permis de voir quels termes étaient associés les uns aux autres : en particulier, à quels acteurs, lieux ou concepts les différents termes étaient les plus connectés.

De façon peu surprenante, dans les discussions sur Internet la distinction entre les usages de ces différents termes est beaucoup plus faible que dans les contextes politique et académique. L'emploi indistinct des termes « migrant » et "refugié ", par exemple, souligne encore une fois le vide catégoriel dans lequel se trouve aujourd'hui le débat sur les migrations liées à l'environnement. Au-delà, il convient aussi de s'interroger sur la déconnexion qui existe entre les discours médiatiques qui sont produits sur les migrations et l'environnement, et la réalité empirique de ces migrations. Cette déconnexion a été régulièrement dénoncée par les chercheurs ${ }^{43}$, mais reste très présente dans les débats médiatiques, comme l'illustre l'usage indistinct des termes « migrant » et « réfugié » sur internet.

L'usage d'une méthode inédite, décrite dans cet article, permet de faire apparaître les termes qui généraient la controverse, et d'identifier les termes plus consensuels - en particulier ceux qui contiennent le mot « déplacé ». La multitude des termes utilisés, et les très nombreuses connexions qui existent entre les différents termes et les acteurs, lieux et concepts auxquels ils sont associés, témoignent de la vitalité de la controverse sur le vocabulaire qui caractérise la relation entre environnement et migration. Elles révèlent aussi la réalité polymorphe de ces migrations, et l'impossibilité de les rassembler en une catégorie unique. Le débat sur la catégorisation de ces migrations reste donc plus ouvert que jamais : l'enjeu, demain, sera d'y associer les migrants eux-mêmes, qui restent les grands absents de ces débats.

En effet, si la controverse sur l'usage des termes renvoie à des débats importants sur la conceptualisation des migrations associées à l'environnement et aux réponses politiques à y apporter, elle renvoie aussi à l'image et à la perception de ces migrations, y compris à la manière dont les migrants se per-

43. Gemenne F., "How they became the human face of climate change. Research and policy interactions in the birth of the 'environmental migration' concept”, in Piguet E., Pécoud A., de Guchteneire P. (eds.) Migration and Climate Change, Cambridge et Paris, Cambridge University Press/UNESCO, 2011, pp. 225-259. 
çoivent eux-mêmes. Ceux-ci se sont positionnés à plusieurs reprises sur la manière dont ils souhaitaient être nommés : selon le contexte, le ressenti de leur situation et les avantages qu'ils pensent en tirer, les migrants préféreront une appellation plutôt qu'une autre. Aujourd'hui, les migrants n'ont aucune voix dans les débats qui entourent la manière adéquate de les nommer : le nom qu'on leur donne jouera pourtant un rôle prépondérant dans leur propre perception de leur migration. À ce titre, il est essentiel que leur voix soit entendue. 